\title{
The Use of Anti-Diabetic Drugs in Alzheimer's Disease, New Therapeutic Options and Future Perspective
}

\author{
Osama H. Mohamed Ibrahim ${ }^{1,2}$, Mariame A. Hassan ${ }^{3,4}$ \\ ${ }^{1}$ Department of Pharmacy Practice and Pharmacotherapeutics, College of Pharmacy, University of Sharjah, Sharjah, UAE \\ ${ }^{2}$ Department of Clinical Pharmacy, Faculty of Pharmacy, Cairo University, Cairo, Egypt \\ ${ }^{3}$ Department of Pharmaceutics and Pharmaceutical Technology, College of Pharmacy, University of Sharjah, Sharjah, UAE \\ ${ }^{4}$ Department of Pharmaceutics and Industrial Pharmacy, Faculty of Pharmacy, Cairo University, Cairo, Egypt \\ Email: oibrahim@sharjah.ac.ae
}

How to cite this paper: Ibrahim, O.H.M. and Hassan, M.A. (2018) The Use of Anti-Diabetic Drugs in Alzheimer's Disease, New Therapeutic Options and Future Perspective. Pharmacology \& Pharmacy, 9, 157-174.

https://doi.org/10.4236/pp.2018.96013

Received: May 8, 2018

Accepted: June 19, 2018

Published: June 22, 2018

Copyright $\odot 2018$ by authors and Scientific Research Publishing Inc. This work is licensed under the Creative Commons Attribution International License (CC BY 4.0).

http://creativecommons.org/licenses/by/4.0/

\begin{abstract}
Alzheimer's disease (AD) is a neurodegenerative disorder that is characterized by progressive loss of memory, confusion, inability of speech and decline in the cognitive behavior. It is considered one of the most common forms of dementia. Clinical studies and preclinical data in the last decade proved that $\mathrm{AD}$ and Diabetes mellitus share a pathophysiological pathway, indicating that insulin resistance, oxidative stress and inflammatory response would increase the risks of developing $\mathrm{AD}$ in diabetic patients. This review presents briefly the etiology of $\mathrm{AD}$ and Diabetes, discusses the possible theories about the interplaying risk factors and the mechanism of action of anti-diabetic medications recommended for the treatment of $\mathrm{AD}$. It is concluded that antidiabetics have good potential to improve dementia, especially in earlier AD stages. However, many of the underlying intricate molecular pathways are still unclear and thus thorough future research is required.
\end{abstract}

\section{Keywords}

Alzheimer's Disease, Antidiabetic Medications, Beta Amyloid, Insulin Resistance, Cognitive Behavior, Metformin

\section{Introduction}

Alzheimer's disease (AD) is a chronic neurodegenerative disease that is recognized by cognitive and memory impairment. It most often begins in people over 65 years of age [1]. Being acknowledged as the most common form of dementia, it is considered as one of the massive societal tragedies. $\mathrm{AD}$ is considered the 
sixth leading cause of death in the United States and is the major worldwide public health issue facing the aging population [2]. In 2015, there were approximately 29.8 million people worldwide with $\mathrm{AD}$. Therefore, researchers and scientists have been working recently round-the-clock on understanding the molecular basis of such neuronal dysfunction and memory loss, racing against expectations of exponential increase in the number of cases through the coming years. Despite the efforts, unfortunately, we still lack an effective treatment that is capable of cutting off $\mathrm{AD}$ progression [3]. Pathologically, $\mathrm{AD}$ is characterized by intracellular neurofibrillary tangles and extracellular amyloidal protein deposits contributing to senile plaques [1]. It is not fully understood why the changes that lead to $\mathrm{AD}$ happen, but several theories were believed to be behind it.

\section{1) Beta Amyloid plaques theory:}

The beta amyloid (BA) plaques are usually found in the brain of $\mathrm{AD}$ patients and are composed of BA, a natural cleavage enzyme that consists of $36-43$ amino acids. BA is usually generated from proteolysis of the amyloid precursor protein (APP) cleaved by certain enzymes e.g. (BACE-1), b-SECRETASE AND $\mathrm{y}$-SECRETASE [4]. BA is involved in neurological survival and protein expression, and any abnormality in the balance of its production or clearance can result in its accumulation outside the neurons forming plaques that interfere with the communication between neurons at the synapses. This may contribute ultimately to neuronal loss and cell death manifesting as memory loss. Today novel animal and clinical studies demonstrate that diabetes is too related to BA pathology, therefore; including diabetes management therapeutics is expected to allow for the development of strategies aiding in the prevention of $\mathrm{AD}$ [5].

\section{2) Tau tangles theory:}

Tau is a microtubule protein of a mature neuron, normally found in the brain. Tau protein provides stabilization to microtubules and gives structural support for the axonal neurons. Hyperphosphorylation of tau protein will form tangles known as Neurofibrillary tangles (NFT) [6] [7]. The NFT play a major role in $\mathrm{AD}$ pathology and further abnormality or mutation in tau protein would lead to neurotoxicity and aggravation of $\mathrm{AD}$.

\section{3) Genetic mutations theory:}

It's rare that $\mathrm{AD}$ develops due to genetic mutations (not more than $1 \%$ incidence) [8]. However, mutations in genes such as amyloid precursor protein (APP), presenilin 1 or presenilin 2 will result in $\mathrm{AD}$, especially if the inheritance of mutation was for both APP and presenilin concomitantly. It is reported that individuals having mutations in these three genes tend to develop AD even before the age of 65 and can be as early as 30 years of age [9].

\section{4) Cholinergic theory:}

It's the oldest theory about $\mathrm{AD}$ that ascribes the etiology of $\mathrm{AD}$ to a decrease in the production of acetylcholine (Ach) in the brain. This theory has been always the basis for treatment strategies, however, it was consistently observed that medications increasing Ach did relieve some of $\mathrm{AD}$ symptoms but the overall therapeutic benefit was not a payoff [10]. 
Stages of $\mathrm{AD} . \mathrm{AD}$ is divided into three stages including: 1) Early stage: Patients have their own work and social life, and can function in a proper way, however; they start to forget things such as coming up with the right words or names when they are introduced to new people or locations, losing valuable objects, and difficulty in organizing or planning. 2) Middle cognitive (moderate) stage: This is the longest stage lasting for many years in most cases. In this stage, appreciable damage occurs to the nerve cells resulting in states of confusion, frustration, anger, mood swinging as well as changes in the sleeping cycle patterns. Progressively, patients become unable to remember specific events or to recall their past lives, e.g. phone numbers and college graduation. They would experience some delusional thoughts repetitively with increased wandering and feeling lost. On the level of cognition-related physical activities, they show difficulty in controlling bowel and bladder movements and in deciding on wearing the appropriate clothes for different seasons. Thus, the patients at this phase would require special care on daily basis. 3) Late (severe) stage: Patients in their late $\mathrm{AD}$ phase are unable to respond to the surrounding environment and they become more vulnerable for increased risk of serious infections, such as pneumonia [11]. They lose ability to talk, respond to a conversation or even walk. Eventually, they lose the ability to swallow. Patients at this stage grow more dependent on assistance around the clock.

Diagnosis of $\mathrm{AD}$. There is no specific test for the diagnosis of $\mathrm{AD}$, however; variety of approaches is usually adopted to confirm its diagnosis. These approaches include:

1) Reporting and evaluating the medical and family history for any psychiatric, cognitive or behavioral condition.

2) Conducting cognitive, physical, or neurological examinations for the patient.

3) Performing comprehensive medical evaluation by a physician.

4) Asking family members through a questionnaire to describe the patient's behavior and cognitive skills.

5) Performing blood tests and brain imaging to rule out other cognitive neurological disorders [12].

Risk factors of AD. Like any other disease, scientists believe that AD can develop due to multiple factors. These factors include Age: Aging is the greatest risk factor for $\mathrm{AD}$. The majority of $\mathrm{AD}$ patients develop the disease at an age of 65 years or older [13]; Family history. First degree relatives, e.g. a parent or a sibling, all contribute to having higher risk of developing the disease especially with the shared environment and lifestyle factors [12]; APOE E4: It is a protein responsible for carrying cholesterol in the bloodstream. Normal individuals may inherit up to three forms of that gene whether E2; E3; or E4. From each parent, E3 is the most common to be inherited (up to 90\%), followed by E4 then lastly E2. E4 increases the risk of developing AD if it was inherited as one copy from one parent, however, risk can increase from 8 to 12 folds if $\mathrm{E} 4$ was inherited from two copies one from each parent [12] [14]. 
Modifiable risk factors. These are risk factors that can be changed through lifetime. They can be modified to reduce the likelihood of having AD.

Cardiovascular conditions. The brain is the most important organ of the body controlling all human activities, yet, its function depends on the heart where $20 \%$ of the body's oxygen supply reaches the brain from the heart. Many factors that increase the risk of cardiovascular problems can result in a decline in cognitive behavior and thus increase the risk of dementia (e.g. diabetes, obesity and smoking). Studies show that impaired glucose metabolism in the brain can increase the risk of dementia [15]. Hypertension and high blood cholesterol levels are well associated with $\mathrm{AD}$. Today, there is strong evidence that healthy diet, physical activity and a lifelong learning cognitive training can also reduce $\mathrm{AD}$ or even prevent it [16].

Education. Interestingly, research has found that people with more years of formal education are at lower risk for $\mathrm{AD}$ as well as other forms of dementia compared to others with fewer years of formal education [17]. It is acknowledged that with more years of education, the transmission of signals between the neurons increases, decreasing the number of areas of the brain that are not being mentally stimulated. Moreover, education impacts economic lifestyle positively: leading more affordable life, having proper healthy diet, and better health care and accessibility to medical treatment [12].

Social engagement. Socially active and engagement with people may reserve the cognitive behavior and support the brain health; however, more research is needed to understand the exact mechanism behind it [12].

Treatment of AD. Until now there is no cure for $\mathrm{AD}$. The available treatments offer only some symptomatic relief and are classified into non-pharmacological and pharmacological therapies.

Non-pharmacological therapy: This includes memory training, listening and remembering the patient's favorite music, aerobic and non-aerobic exercises. These practices can delay the decline in the cognitive behavior, reduce the behavioral symptoms and improve the quality of life [18] [19].

Pharmacological therapy: The current medications used nowadays are: Donepezil. It is a short acting acetylcholinesterase inhibitor used for all stages of AD. It inhibits the degradation of acetylcholine in the synaptic cleft increasing its reuptake and, thus, it enhances the cholinergic neurotransmission [20]. Galantamine. It is used for mild to moderate $\mathrm{AD}$. It is a competitive and reversible acetylcholinesterase inhibitor, and an allosteric modulator at nicotinic cholinergic receptor sites and, hence, it enhances the cholinergic nicotinic neurotransmission [21] [22]. Memantine. It is non-competitive N-methyl-d-aspartate (NMDA) receptor blocker used for moderate to severe AD. It was found that targeting glutamatergic system specifically NMDA receptors could be beneficial in the management of Alzheimer's because the impairment of glutamatergic pathway usually leads to neuronal toxicity [23]. Rivastigmine. This is also a cholinesterase inhibitor used to enhance cholinergic stimulation [24]. 
$\mathrm{AD}$ and Diabetes. Both $\mathrm{AD}$ and Diabetes are more prevalent in adults and the risk of their incidences increases with aging. They do have a strikingly shared pathophysiology that some researchers refer to AD as Type 3 Diabetes [25] [26].

Diabetes mellitus is a chronic metabolic condition which affects our body's ability to metabolize glucose. It's characterized by hyperglycemia which occurs as a result of decreased effectiveness of Insulin. There are three major types of diabetes: type I diabetes; type II diabetes; and gestational diabetes.

Type I diabetes is an autoimmune condition in which the immune system attacks and kills the beta cells located in the pancreas and which are responsible for insulin production. The disease usually occurs during childhood, but can still develop during adolescence and adulthood. Patients with type I diabetes are mostly well -treated by having their blood glucose levels controlled by daily intake of insulin.

Type II diabetes develops when the body does not use insulin efficiently; i.e. when the body becomes resistant to insulin and the uptake of glucose into cells is suppressed. This type represents $85 \%-90 \%$ of all cases of diabetes. Patients of this type are usually treated by lifestyle modifications and weight reduction plans, besides oral hypoglycemic agents [27].

Gestational diabetes develops in pregnant women when cortisol, estrogen and lactogen produced by the placenta can block insulin resulting in insulin resistance. Gestational diabetes usually subsides after delivery [28].

Uncontrolled diabetes may lead to long-term complications, such as nerve damage, vision problems and, most importantly, heart diseases and nephropathy. Due to the impairment of all vital organs including the brain, AD is now listed as one of the complications of diabetes [29]. The relationship between AD and diabetes could be simply explained by the damage caused by diabetes to the blood vessels feeding the nerve cells (microvascular and macrovascular changes). This damage results in impairment of neurons and ultimately their death [30]. Also, blood glucose levels and insulin affect the brain and vice versa [31]. Insulin is known to affect different brain functions including attention, learning, memory, and cognition. Therefore, insulin resistance or deficiency may play a huge role in $\mathrm{AD}$. Targeting insulin to reduce the risk of $\mathrm{AD}$ could thus be a great opportunity for people who are at risk of developing $\mathrm{AD}$ during their life courses. In what follows, many interesting theories that relate diabetes to $\mathrm{AD}$ will be discussed. The aim is to explore the possibility of using anti-diabetic medications in the prevention and slowing the progression of $\mathrm{AD}$.

\section{The Connection between Alzheimer's Disease and Diabetes}

According to some systematic reviews and epidemiological data as well as upon the examination of individuals with diabetes, it was found that they have an increased risk of dementia specifically $\mathrm{AD}$ and vascular dementia when compared to those without diabetes [32]. An updated meta-analysis of cohort study that included 17 studies involving 1,746,777 individuals discussed the risk of devel- 
oping $\mathrm{AD}$ among diabetic patients. The results proved that patients with diabetes have a higher incidence of developing $A D$ than those without diabetes [33]. Patients with diabetes and APOE4-the strongest risk factor of AD, they are two folds more prone to develop $\mathrm{AD}$ than others [34]. The molecular relationship between $\mathrm{AD}$ and Diabetes is complex. There are many theories trying to explain this intricate association through insulin resistance, oxidative stress, inflammatory response, acetylcholine, BA or obesity. Many published reports have concluded that $\mathrm{AD}$ can be considered as a metabolic disease in which the brain loses the ability to consume and utilize glucose due to insulin resistance [35].

Insulin activity in brain and the effect of insulin resistance on cognitive behavior. In spite of the fact that insulin plays a significant role on brain functions including cognition, attention and memory, unfortunately, the majority of people are not aware of the effects of insulin on the central nervous system. Insulin can traverse the blood brain barrier (BBB) by active transport into the brain where insulin receptors are omnipresent on nerve cells [7]. It is also believed that insulin may be produced locally in the brain [36]. Stimulation of transporter proteins that enhance the influx of glucose usually occurs due to the binding of insulin to dimerized receptors. The fact that neurons can still obtain glucose in different mechanisms that do not depend on insulin explains the fact that insulin receptors are distributed unequally throughout the brain. The early stages of Diabetes type II is defined by insulin resistance and hyperinsulinemia (pancreatic overproduction of insulin). Acute hyperinsulinemia may have good impact on brain functions including cognition and memory. However, prolonged high levels of insulin may, contrarily, exert a negative effect on cognition and memory [37]. Increased peripheral insulin levels may further raise the levels of insulin in cerebrospinal fluid and brain. These sustained high levels of insulin will result in down regulation of insulin receptors in $\mathrm{BBB}$ and a consequent decrease in insulin transport into the brain [7]. Clinical observations of $\mathrm{AD}$ patients revealed that in mild $\mathrm{AD}$, induced hyperglycemia may lead to raised plasma insulin level and improved cognitive performance. These effects of hyperglycemia do not occur in patients with severe or late stage AD [38]. Disruption of cerebral insulin receptors by administration of streptozotocin (STZ) injection, a diabetogenic toxin, by intracerebroventricular route into a group of rats led to inadequate energy metabolism and reduced cognitive behavior and performance [2] [18]. Moreover, it has been reported that despite the increased expression of insulin receptors in patients with sporadic $\mathrm{AD}$, there was a decrease in the activity of insulin receptors-mediated tyrosine kinase, the thing that indicates the impairment of insulin signal transduction [39].

Oxidative Stress. A free radical is an oxygen/nitrogen atom with one or more unpaired electrons that makes it highly reactive to have interactions with other molecules. Reactive oxygen species (ROS) are cytotoxic products of normal mitochondrial metabolism that are normally cleared by antioxidant pathways in the living systems [37] [40]. In brain, ROS can be either beneficial or harmful; i.e. they have a dual biological roles. Low levels of ROS produce a beneficial role 
in cellular responses and synaptic signaling, while moderate levels of ROS are believed to enhance the peripheral insulin resistance [37] [40]. However, when the metabolic activity of cells exceeds their anti-oxidant capacity due to imbalance in mitochondrial ROS production, a state of oxidative stress (OS) results, which is normally followed by cell damage and finally cell death [37]. OS and mitochondrial dysfunction play an important role in the pathological processes of both $\mathrm{AD}$ and Diabetes as evident by the high levels of ROS and reactive nitrogen species (RNS) detected in both diabetic and AD patients [14]. ATP required for the normal function of cells and neurons is mainly provided by mitochondria, thus, mitochondrial impairment can result in cellular and neuronal damage [6]. In fact, the brain is extremely sensitive to OS due to high requirements of oxygen, and along with a deficiency in the anti-oxidant system, the damage can be aggravated. Thus, it has been shown that an increase in OS can lead to further inhibition of the production of cellular energy and reduction in both insulin secretion and insulin-receptor sensitivity. Meanwhile, the impaired insulin signaling makes the neurons energy-deficient and increases their susceptibility to oxidation injury. This, in turn, might worsen the structural and functional deterioration of mitochondria; i.e. a vicious cycle ensues [18]. OS also results in the production of advanced glycation end products (AGEs). The receptors of advanced glycation end products (RAGE) will interact with BA peptides and exacerbate the neuronal stress and inflammation which will result in impaired cognitive abilities [40] [41].

Inflammatory Response. Insulin resistance, the main characteristic of type II diabetes, is associated with increased level of inflammatory mediators such as interleukin-6 (IL-6) and C-reactive protein (CRP). The high levels of these inflammatory substances are linked to immunological impairment which results in insulin resistance [42]. Additionally, there is sufficient evidence that $\mathrm{AD}$ is associated with inflammatory processes [43] [44]. IL-6 was found in the senile plaques of patients' brains as well as in their lumbar and ventricular cerebrospinal fluid [6]. The inflammatory products accumulate in the brain of $\mathrm{AD}$ patient at different rates compared to healthy subjects. It is suggested that CRP can be linked to increased risk of $\mathrm{AD}$ in some studies [45] while the prolonged use of non-steroidal anti-inflammatory drugs (NSAIDs) for chronic pain management can reduce the chances of getting $\mathrm{AD}$ [6]. Therefore, it can conclude that inflammation is a common process in both Diabetes and $\mathrm{AD}$, whether causative or consequential, and thus they bear a shared pathophysiology.

Acetylcholine. $\mathrm{AD}$ is characterized by a vast suppression in choline acetyl transferase (ChAT) and a reduction in ChAT immunoreactivity with insulin and Insulin-like growth factor-1 (IGF-1) receptors in cortical neurons [45]. According to experimental data, insulin and IGF-1 receptors can control Tau protein and ChAT gene expression [46].

Tau protein and BA. Insulin and BA are produced by beta cells in pancreas. The elevation in BA is reported to affect negatively the synaptic plasticity [47]. Diabetic patients have been observed with BA plaques which interfere with the 
signaling pathways in the brain phosphorylating the tau protein and forming aggregated neurofibrillary tangles. Several studies showed that patients who suffer from Diabetes mellitus may develop hyper-phosphorylation of tau protein and abnormal clearance of BA-which are the main characteristics of $\mathrm{AD}$ - in a higher rate compared to a non-diabetic patient [45]. Several mechanisms have been introduced to explain the reasons underlying the phosphorylation of tau. The most significant proposed mechanism is the impaired insulin signaling pathway that causes a decrease in phosphatidylinositol-3-kinase (PI3K), Protein kinase B (AKT), and increase in the glycogen synthase kinase (GSK). Other studies suggest that insulin can alter the kinases that regulate tau, including c-Jun N-terminal Kinase (JNK), mitogen-activated protein kinase (P38 MAPK) [48] [49]. On the other hand, tau phosphorylation is considered as one of the biggest theories that contribute to $\mathrm{AD}$ development and progression resulting in tangles, plaques that can lead to impaired axonal neuronal transport, destabilization and memory loss [50]. Thus, tau phosphorylation and the formed tangles can be considered as the link between both diabetes and AD [51]. JNK is considered one of the key players that contribute to both OS and tau phosphorylation. JNK is known as the islets brain and was observed at chronic stages of insulin resistance and hyperglycemia as it induces apoptosis in beta cells [52].

Obesity. Obesity can affect glucose metabolism, increase insulin resistance and lead to hypertension and dyslipidemia which are all factors that contribute to type II diabetes [53]. A high body mass index can affect insulin pathway and cause disturbance in the liver and the muscle cells through the pro-inflammatory response [54]. A population study shows that as the body mass index increase $1 \%$ at the age of 70 , the risk of developing $\mathrm{AD}$ increase by $36 \%$ [55]. There are lots of studies and reports that suggest the correlation between the cognitive behavior, the motor function of the brain and obesity indicating that the deterioration of cognitive function can increase with high body mass index regardless of the fat distribution. In fact, a cohort study of obese women has been done and results indicated that the increase in their body weight was associated with derangement of their motor function [56].

\section{Classes of Anti-Diabetic Drugs as Potential Treatments for Alzheimer's Disease}

Based on the shared pathophysiology between diabetes and AD, several studies have been conducted to assess the neuroprotective effect of some anti-diabetic medications and their mechanism of action. Currently, some anti-diabetic drugs are approved to be incorporated in the treatment of $\mathrm{AD}$, and many are yet under investigation in clinical trials [57].

Insulin. Preclinical studies in rat model show that the intra-cerebroventricular STZ injection induced cognitive dysfunction, while the intraventricular administration of Detemir, a long-acting insulin analog, was able to correct the cognitive decline as manifested by the considerable increase in learning ability [58]. Moreover, many clinical studies have pointed that hyperinsulinemia without 
hyperglycemia enhances memory in adults with $\mathrm{AD}$ indicating an important role of this hormone in memory facilitation [57]. The systemic administration of insulin is linked to increased risk of hypoglycemia and reduced penetration of insulin into the central nervous system. These issues were overcome by introducing intranasal administration of insulin to directly target the brain. Preclinical evidence showed that intranasal administration of insulin counteracts the protective action $\mathrm{BBB}$, and in human studies, after intranasal administration a significant amount of insulin arrived the brain in an active state. A study conducted on healthy volunteers showed that the intranasal administration of insulin $(4 \times$ $40 \mathrm{IU} / \mathrm{d}$ ) for 8 weeks resulted in improved mood and word recall [57]. It was found that in patients with early $\mathrm{AD}$ and mild cognitive impairment (MCI), acute intranasal administration of 20 or $40 \mathrm{IU} / \mathrm{d}$ of insulin showed an improvement in memory. However, a poorer word recall was observed in memory-impaired subjects carrying the APOEe4 allele in comparison to patients who do not possess this allele and the healthy controls. This points out that APOE might play a role in mediating insulin effects in the central nervous system. Further studies on the same group suggested that there is a differential dose-response curve to intranasal insulin such that functional status, verbal memory and attention was improved by a relatively low dose of insulin in patients that lack the APOEe4 allele; however, in patients with APOEe4 there was a relative decline in memory [59].

Thiazolidinediones. These are famous class of anti-diabetic medications that are agonists of the peroxisome proliferator-activated receptor-gamma (PPAR $\gamma$ ). They work by increasing the response of body to insulin, thus, decreasing plasma glucose level and insulin resistance [60]. The safety of Rosiglitazone has limited its use in the management of diabetes since it increases the risk of cardiovascular events. As a result, Pioglitazone is the only drug in this class used nowadays in Diabetes management. The conceptbehind testing this group of medications in AD is mainly because of the over expression of PPAR $\gamma$ in the temporal cortex compared to healthy brains. $\operatorname{PAAR} \gamma$ can enhance cognitive behavior by different mechanisms involving the enhancement of BA clearance, reduction of $\beta$-secretase and APP expression but not APP degradation [61]. In preclinical studies, PPAR $\gamma$ agonists have shown the ability to reduce BA plaque formation and inflammatory genes [57]. They can also modulate calcium homeostasis in the hippocampus and provide neuroprotective effect [62]. In a pilot study performed on patients with $\mathrm{AD}$ and mild cognitive impairment, the results showed that 6-months treatment with Rosiglitazone was able to improve memory and attention when compared to placebo. On the other hand, in a phase III clinical trial on AD patients, Rosiglita zone as adjunctive therapy to acetylcholine esterase inhibitors failed to improve or enhance memory and cognition [63]. Another pilot study was performed on $\mathrm{AD}$ patients who suffer from diabetes; they were given Pioglitazone 15 - $30 \mathrm{mg}$ for 6 months. The results observed were enhanced cognition and increased cerebral blood flow compared to controls. No efficacy observed when Pioglitazone was given for 18 months to patients who 
suffered from $\mathrm{AD}$ without diabetes, although it was well tolerated. A very recent meta-analysis on PPAR $\gamma$ agonists indicates that Pioglitazone is the only drug that can improve memory and cognition behavior in early stages of the disease or in mild to moderate $\mathrm{AD}$ [64].

Sulfonylurea. The inhibition of ATP-sensitive $\mathrm{K}^{+}$channels (KATP) channel of pancreatic beta cells by Sulfonylureas stimulate insulin secretion and increase its levels in the blood, therefore, it lowers blood glucose. Glibenclamide (1 $\mathrm{mg} / \mathrm{kg}$ ) when administered intraperitoneally to rats that had undergone ischemic-reperfusion injury, an increase in glutathione levels and total anti-oxidant capacity accompanied with decreased lipid peroxidation were observed emphasizing its potential use in modulating OS and inflammatory mediators [37]. In a study was performed to assess the effectiveness of gliclazide in protecting diabetes-induced OS in the brain of diabetic rats, it was shown that there was an increase in the total anti-oxidant defense and a decrease in total oxidant index in the brains [65].

Metformin. It is considered one of the most common anti-diabetic medications for type II diabetes that has been studied extensively and reported to possess promising outcomes compared to the other medications. It belongs to biguanides group that acts by increasing insulin in the peripheral tissues, reducing serum glucose and suppressing hepatic gluconeogenesis. It is the only oral drug used for the treatment of diabetes that does not cause weight gain, however, it should be used cautiously with patients suffering from hepatic and renal failure [66]. Metformin can be considered one of the most controversial medications for its own mechanism of action and its relation to dementia and AD. Some clinical trials reported that the long-term treatment with metformin could result in lower risk of cognitive impairment. It can influence the anti-inflammatory, anti-oxidative and anti-apoptotic pathways in patients by decreasing the interleukin $1 \beta$ (IL-1 $\beta$ )-induced activation of pro-inflammatory phosphokinases Akt and P38 MAPK involved inAD etiology [67]. Metformin acts by inhibiting mitochondrial respiratory chain complex 1 , glucagon-induced elevation of cyclic adenosine monophosphate (cAMP), activation of both protein kinase A (PKA) and activated protein kinase (AMPK) [68] [69]. The activation of AMPK pathway provides neuro-protection and exerts a positive effect on human neural stem cells. AMPK plays an important role in the regulation of intracellular event such as glucose uptake, metabolism of lipids and mitochondrial biogenesis. Since there's a connection between $\mathrm{AD}$ pathogenesis and cellular mechanisms of energy homeostasis, targeting AMPK pathway in human neural stem cells could be a therapeutic option for $\mathrm{AD}$ [70]. The ability of metformin to decrease the development of AD was studied through one-year administration of metformin to APP transgenic mice, which over express mutant APP and serve as model for $\mathrm{AD}$. The results showed that the effect was gender-dependent where the activation of AMPK could improve memory and learning in female mice. On the other hand, male mice receiving the same treatment showed worsening of memory and cognitive behavior. Such results can be explained on hormonal bases in 
which the hormonal signaling in females was enhanced by metformin [71]. Clinical studies have been carried out to investigate the effect of different anti-diabetic medications given to participants for a period of 4 years. It was shown that only participants who took metformin had improvement in their cognitive behavior including memory and verbal learning [72]. In another study, the influence of metformin on scopolamine-associated memory loss in wistar rats was investigated. Rats were administered different doses of metformin $(100 \mathrm{mg} / \mathrm{kg}$ and $300 \mathrm{mg} / \mathrm{kg}$ ). Only the lower dose showed a significant retardation in memory loss possibly due to a suppression of the elevated AKT and phosphorylated tau [73]. Another study was performed to investigate the effect of metformin on memory enhancement that was conducted for 6 weeks. The results further supported the ability of metformin to improve mice memory. The process included recovery of long-term potentiation (LTP) and normalization of several brain molecular alterations including the receptor that is responsible of RAGE [30]. The study by Hettich et al. states that metformin can reduce BACE1 protein expression in vivo tests and in cell culture models, leading to a decrease in BACE1 cleavage products and reducing the production of BA [74]. Other studies proposed that the mechanism of action of metformin might be attributed to anti-oxidant activity [75]. On the other hand, researchers negated any role of metformin on the Ach receptor when the anti-diabetic canagliflozin and metformin were used in rat model and data showed a decrease in ach activity with canaglifozin but not with metformin after two weeks [76]. Metforminis able to decrease the serum lipids, through decreasing inflamed cells adherence to the blood vessels in the endothelium, preventing hyperlipidemia, cholesterolemia and development of cardiovascular complication. Considering the potential of metformin in $\mathrm{AD}$, this might augment the arguments that diabetes may not be linked to $\mathrm{AD}$ in spite of the salient statistical evidence [77] [78] [79]. However, cardiovascular problems are known to be one of the biggest risk factors for developing AD. In a recent clinical trial of 355 patients diagnosed with stroke and diabetes, patients were administered metformin prior to their stroke onset and the results showed an association with reduced neurological severity [80]. The exact mechanism of metformin action on cardiovascular system is not fully understood until now, but it was suggested that metformin could enhance the myocardial preconditioning during ischemia, protect tissues from loss of oxygen and prevent cell apoptosis and development of heart failure [81]. On the other hand, metformin and sulfonylurea were given to patients who did not receive any anti-diabetic treatment and the two medications greatly decreased the onset of dementia [82]. In contrast, when metformin was given alone, the risk of developing dementia and $\mathrm{AD}$ increased and the investigators concluded that metformin as single therapeutic agent did not show an effect alone in decreasing memory loss [83]. The administration of metformin for 24 weeks to depressed patients with type II diabetes resulted in improved cognitive behavior, yet, metformin exerted no effect on patients with depression and APOE- $\varepsilon 4$ [84]. McNeily investigated the ability of metformin to improve cognitive damage induced by high fat diet. The results 
revealed that while metformin can decrease insulin resistance and weight gain associated with diet, it had no influence on short-term memory or cognitive alterations [85]. Therefore, it is obvious from this discussion that despite the extensive efforts on metformin, there are lots of contradictory data emerging from the clinical trials conducted about metformin drug therapy, especially those relating $\mathrm{AD}$ with diabetes.

Amylin Analogue. Amylin is released with insulin from the beta pancreatic cells in response to glucose intake. It is able to regulate the glucose level and slow the gastric emptying rate [86]. Its analogue pramlintide has been approved as a medication for the treatment of both type I and type II diabetes. Amylin plasma concentration in $\mathrm{AD}$ patients was found to be low and has the ability to cross the $\mathrm{BBB}$ and modulate the memory, mood and anxiety [87]. Administration of amylin analogue pramlintide in preclinical data of $\mathrm{AD}$ mouse models was found to reduce OS, neuro-inflammation and enhance the memory [88].

\section{Conclusion}

Diabetic patients are at more risk to develop $\mathrm{AD}$ than healthy individuals. $\mathrm{AD}$ and diabetes share a common pathophysiology through insulin resistance, OS, inflammatory response, BA and tau phosphorylation. The previously mentioned theories can all occur and contribute to both diabetes and AD. Therefore, researchers have been studying the effect of anti-diabetic medications on the prevention or slowing down the progression of $\mathrm{AD}$. In this review, the results of various studies were presented and discussed on whether the anti-diabetic medications can actually exert an effect on brain functions including memory, cognition and attention. Intranasal administration was considered as the best route for insulin delivery into the brain as it traverses directly into the BBB. Metformin is considered one of the most important oral medications given in diabetic patients, except for those who suffer from lactic acidosis, and has been studied extensively. There are many clinical studies tend to support its efficacy in cognitive enhancement. Despite controversial findings, it is agreed that the earlier the stage of $\mathrm{AD}$, the more chance for anti-diabetics to have positive effect on brain functions. More clinical trials and studies should be done in this field to limit the progression of $\mathrm{AD}$, find better treatment outcomes and increase the quality of life in $\mathrm{AD}$ patients.

\section{References}

[1] Pardeshi, R., et al. (2017) Insulin Signaling: An Opportunistic Target to Minify the Risk of Alzheimer's Disease. Psychoneuroendocrinology, 83, 159-171. https://doi.org/10.1016/j.psyneuen.2017.05.004

[2] de la Monte, S.M. and Wands, J.R. (2008) Alzheimer's Disease Is Type 3 Diabetes-Evidence Reviewed. Journal of Diabetes Science and Technology, 2, 1101-1113. https://doi.org/10.1177/193229680800200619

[3] De Felice, F.G., Lourenco, M.V. and Ferreira, S.T. (2014) How Does Brain Insulin Resistance Develop in Alzheimer's Disease? Alzheimer's \& Dementia, 10, S26-32. 
https://doi.org/10.1016/j.jalz.2013.12.004

[4] LaFerla, F.M., Green, K.N. and Oddo, S. (2007) Intracellular Amyloid-Beta in Alzheimer's Disease. Nature Reviews. Neuroscience, 8, 499-509.

https://doi.org/10.1038/nrn2168

[5] Hardy, J. and Selkoe, D.J. (2002) The Amyloid Hypothesis of Alzheimer's Disease: Progress and Problems on the Road to Therapeutics. Science, 297, 353-356. https://doi.org/10.1126/science.1072994

[6] Akter, K., et al. (2011) Diabetes Mellitus and Alzheimer's Disease: Shared Pathology and Treatment? British Journal of Clinical Pharmacology, 71, 365-376. https://doi.org/10.1111/j.1365-2125.2010.03830.x

[7] Gasparini, L., et al. (2002) Does Insulin Dysfunction Play a Role in Alzheimer's Disease? Trends in Pharmacological Sciences, 23, 288-293. https://doi.org/10.1016/S0165-6147(02)02037-0

[8] Bekris, L.M., et al. (2010) Genetics of Alzheimer Disease. Journal of Geriatric Psychiatry and Neurology, 23, 213-227. https://doi.org/10.1177/0891988710383571

[9] Goldman, J.S., et al. (2011) Genetic Counseling and Testing for Alzheimer Disease: Joint Practice Guidelines of the American College of Medical Genetics and the National Society of Genetic Counselors. Genetics in Medicine, 13, 597-605. https://doi.org/10.1097/GIM.0b013e31821d69b8

[10] Francis, P.T., et al. (1999) The Cholinergic Hypothesis of Alzheimer's Disease: $A$ Review of Progress. Journal of Neurology, Neurosurgery, and Psychiatry, 66, 137-147. https://doi.org/10.1136/jnnp.66.2.137

[11] Forstl, H. and Kurz, A. (1999) Clinical Features of Alzheimer's Disease. European Archives of Psychiatry and Clinical Neuroscience, 249, 288-290. https://doi.org/10.1007/s004060050101

[12] Association, A.S. (2017) Alzheimer's Association Report: 2017 Alzheimer's Disease Facts and Figures. Alzheimer's \& Dementia, 13, 325-373. https://doi.org/10.1016/j.jalz.2017.02.001

[13] Hebert, L.E., et al. (2010) Change in Risk of Alzheimer Disease over Time. Neurology, 75, 786-791. https://doi.org/10.1212/WNL.0b013e3181f0754f

[14] Baglietto-Vargas, D., et al. (2016) Diabetes and Alzheimer's Disease Crosstalk. Neuroscience \& Biobehavioral Reviews, 64, 272-287. https://doi.org/10.1016/j.neubiorev.2016.03.005

[15] Ma, H.R., et al. (2018) Cerebral Glucose Metabolic Prediction from Amnestic Mild Cognitive Impairment to Alzheimer's Dementia: A Meta-Analysis. Translational Neurodegeneration, 7, 9. https://doi.org/10.1186/s40035-018-0114-Z

[16] Baumgart, M., Snyder, H.M., Carrillo, M.C., Fazio, S., Kim, H. and Johns, H. (2015) Summary of the Evidence on Modifiable Risk Factors for Cognitive Decline and Dementia: A Population-Based Perspective. Alzheimer's \& Dementia, 11, 718-726. https://doi.org/10.1016/j.jalz.2015.05.016

[17] Sando, S.B., et al. (2008) Risk-Reducing Effect of Education in Alzheimer's Disease. International Journal of Geriatric Psychiatry, 23, 1156-1162. https://doi.org/10.1002/gps.2043

[18] Farina, N., Rusted, J. and Tabet, N. (2014) The Effect of Exercise Interventions on Cognitive Outcome in Alzheimer's Disease: A Systematic Review. International Psychogeriatrics, 26, 9-18.

[19] Onieva-Zafra, M. D., Hernández-Garcia, L., Gonzalez-del-Valle, M.T., Parra-Fernández, M.L. and Fernandez-Martinez, E. (2018) Music Intervention with 
Reminiscence Therapy and Reality Orientation for Elderly People with Alzheimer Disease Living in a Nursing Home: A Pilot Study. Holistic Nursing Practice, 32, 43-50. https://doi.org/10.1097/HNP.0000000000000247

[20] Cacabelos, R. (2007) Donepezil in Alzheimer's Disease: From Conventional Trials to Pharmacogenetics. Neuropsychiatric Disease and Treatment, 3, 303-333.

[21] Olin, J. and Schneider, L. (2002) Galantamine for Alzheimer's Disease. The Cochrane Database of Systematic Reviews, Article ID: CD001747.

[22] Loy, C. and Schneider, L. (2006) Galantamine for Alzheimer's Disease and Mild Cognitive Impairment. The Cochrane Database of Systematic Reviews, Article ID: CD001747. https://doi.org/10.1002/14651858.CD001747.pub3

[23] Cacabelos, R., Takeda, M. and Winblad, B. (1999) The Glutamatergic System and Neurodegeneration in Dementia: Preventive Strategies in Alzheimer's Disease. International Journal of Geriatric Psychiatry, 14, 3-47. https://doi.org/10.1002/(SICI)1099-1166(199901)14:1<3::AID-GPS897>3.0.CO;2-7

[24] Furiya, Y., Tomiyama, T., Izumi, T., Ohba, N. and Ueno, S. (2018) Rivastigmine Improves Appetite by Increasing the Plasma Acyl/Des-Acyl Ghrelin Ratio and Cortisol in Alzheimer Disease. Dementia and Geriatric Cognitive Disorders Extra, 8, 77-84. https://doi.org/10.1159/000487358

[25] Eric, S., Benjamin, T.M., Enrique, J.R., et al. (2005) Impaired Insulin and Insulin-Like Growth Factor Expression and Signaling Mechanisms in Alzheimer's Disease-Is This Type 3 Diabetes? Journal of Alzheimer's Disease, 7, 63-80. https://doi.org/10.3233/JAD-2005-7107

[26] Kroner, Z. (2009) The Relationship between Alzheimer's Disease and Diabetes: Type 3 Diabetes? Alternative Medicine Review, 14, 373-379.

[27] Gloyn, A.L. and Drucker, D.J. (2018) Precision Medicine in the Management of Type 2 Diabetes. The Lancet Diabetes \& Endocrinology. https://doi.org/10.1016/S2213-8587(18)30052-4

[28] Toledano, Y., Hadar, E. and Hod, M. (2018) Pharmacotherapy for Hyperglycemia in Pregnancy-The New Insulins. Diabetes Research and Clinical Practice. https://doi.org/10.1016/j.diabres.2018.04.035

[29] Simó, R., Ciudin, A., Simó-Servat, O. and Hernández, C. (2017) Cognitive Impairment and Dementia: A New Emerging Complication of Type 2 Diabetes-The Diabetologist's Perspective. Acta Diabetologica, 54, 417-424.

https://doi.org/10.1007/s00592-017-0970-5

[30] Markowicz-Piasecka, M., Sikora, J., Szydłowska, A., Skupień, A., Mikiciuk-Olasik, E. and Huttunen, K.M. (2017) Metformin-A Future Therapy for Neurodegenerative Diseases: Theme: Drug Discovery, Development and Delivery in Alzheimer's Disease Guest Editor: Davide Brambilla. Pharmaceutical Research, 34, 2614-2627. https://doi.org/10.1007/s11095-017-2199-y

[31] Leloup, C., Allard, C., Carneiro, L., Fioramonti, X., Collins, S. and Pénicaud, L. (2016) Glucose and Hypothalamic Astrocytes: More than a Fueling Role? Neuroscience, 323, 110-120. https://doi.org/10.1016/j.neuroscience.2015.06.007

[32] Biessels, G.J., Staekenborg, S., Brunner, E., Brayne, C. and Scheltens, P. (2006) Risk of Dementia in Diabetes Mellitus: A Systematic Review. The Lancet Neurology, 5, 64-74. https://doi.org/10.1016/S1474-4422(05)70284-2

[33] Zhang, J., Chen, C.X. and Hua, S.Z., et al. (2017) An Updated Meta-Analysis of Cohort Studies: Diabetes and Risk of Alzheimer's Disease. Diabetes Research and Clinical Practice, 124, 41-47. https://doi.org/10.1016/j.diabres.2016.10.024 
[34] Peila, R., Rodriguez, B.L. and Launer, L.J. (2002) Type 2 Diabetes, APOE Gene, and the Risk for Dementia and Related Pathologies: The Honolulu-Asia Aging Study. Diabetes, 51, 1256-1262. https://doi.org/10.2337/diabetes.51.4.1256

[35] de la Monte, S.M. (2014) Type 3 Diabetes Is Sporadic Alzheimer's Disease: Mini-Review. European Neuropsychopharmacology, 24, 1954-1960. https://doi.org/10.1016/j.euroneuro.2014.06.008

[36] Schulingkamp, R.J., Pagano, T.C., Hung, D. and Raffa, R.B. (2000) Insulin Receptors and Insulin Action in the Brain: Review and Clinical Implications. Neuroscience \& Biobehavioral Reviews, 24, 855-872. https://doi.org/10.1016/S0149-7634(00)00040-3

[37] Butterfield, D.A., Domenico, F.D. and Barone, E. (2014) Elevated Risk of Type 2 Diabetes for Development of Alzheimer Disease: A Key Role for Oxidative Stress in Brain. Biochimica et Biophysica Acta (BBA)-Molecular Basis of Disease, 1842, 1693-1706. https://doi.org/10.1016/j.bbadis.2014.06.010

[38] Suzanne, C., et al. (1993) Effects of Hyperglycemia on Memory and Hormone Levels in Dementia of the Alzheimer Type: A Longitudinal Study. Behavioral Neuroscience, 107, 926-940. https://doi.org/10.1037/0735-7044.107.6.926

[39] Frölich, L., et al. (1998) Brain Insulin and Insulin Receptors in Aging and Sporadic Alzheimer's Disease. Journal of Neural Transmission, 105, 423-438. https://doi.org/10.1007/s007020050068

[40] de la Monte, S.M. and Tong, M. (2014) Brain Metabolic Dysfunction at the Core of Alzheimer's Disease. Biochemical Pharmacology, 88, 548-549.

https://doi.org/10.1016/j.bcp.2013.12.012

[41] de la Monte, S.M. (2009) Insulin Resistance and Alzheimer's Disease. BMB Reports, 42, 475-481. https://doi.org/10.5483/BMBRep.2009.42.8.475

[42] Kandimalla, R., Thirumala, V. and Reddy, P.H. (2017) Is Alzheimer's Disease a Type 3 Diabetes? A Critical Appraisal. Biochimica et Biophysica Acta (BBA)-Molecular Basis of Disease, 1863, 1078-1089. https://doi.org/10.1016/j.bbadis.2016.08.018

[43] Akiyama, H., et al. (2000) Inflammation and Alzheimer's Disease. Neurobiology of Aging, 21, 383-421. https://doi.org/10.1016/S0197-4580(00)00124-X

[44] Rogers, J. and Shen, Y. (2000) A Perspective on Inflammation in Alzheimer's Disease. Annals of the New York Academy of Sciences, 924, 132-135. https://doi.org/10.1111/j.1749-6632.2000.tb05571.x

[45] Mittal, K. and Katare, D.P. (2016) Shared Links between Type 2 Diabetes Mellitus and Alzheimer's Disease: A Review. Diabetes \& Metabolic Syndrome: Clinical Research \& Reviews, 10, S144-S149. https://doi.org/10.1016/j.dsx.2016.01.021

[46] de la Monte, S.M., Ming, T., Nataniel, L.-C., Michael P.J. and Wands, J.R. (2006) Therapeutic Rescue of Neurodegeneration in Experimental Type 3 Diabetes: Relevance to Alzheimer's Disease. Journal of Alzheimer's Disease, 10, 89-109. https://doi.org/10.3233/JAD-2006-10113

[47] Akhtar, M.W., et al. (2016) Elevated Glucose and Oligomeric $\beta$-Amyloid Disrupt Synapses via a Common Pathway of Aberrant Protein S-Nitrosylation. Nature Communications, 7, Article ID: 10242. https://doi.org/10.1038/ncomms10242

[48] Clodfelder-Miller, B.J., Zmijewska, A.A., Johnson, G.V.W. and Jope, R.S. (2006) Tau Is Hyperphosphorylated at Multiple Sites in Mouse Brain in Vivo after Streptozotocin-Induced Insulin Deficiency. Diabetes, 55, 3320-3325. https://doi.org/10.2337/db06-0485 
[49] de la Monte, S.M. (2012) Brain Insulin Resistance and Deficiency as Therapeutic Targets in Alzheimer's Disease. Current Alzheimer Research, 9, 35-66. https://doi.org/10.2174/156720512799015037

[50] Spires-Jones, T.L., Stoothoff, W.H., de Calignon, A., Jones, P.B. and Hyman, B.T. (2009) Tau Pathophysiology in Neurodegeneration: A Tangled Issue. Trends in Neurosciences, 32, 150-159. https://doi.org/10.1016/j.tins.2008.11.007

[51] Abbondante, S., Baglietto-Vargas, D., Rodriguez-Ortiz, C.J., Estrada-Hernandez, T., Medeiros, R. and LaFerla, F.M. (2014) Genetic Ablation of Tau Mitigates Cognitive Impairment Induced by Type 1 Diabetes. American Journal of Pathology, 184, 819-826. https://doi.org/10.1016/j.ajpath.2013.11.021

[52] Thakur, A., Wang, X.L., Siedlak, S.L., Perry, G., Smith, M.A. and Zhu, X.W. (2007) C-Jun Phosphorylation in Alzheimer Disease. Journal of Neuroscience Research, 85, 1668-1673. https://doi.org/10.1002/jnr.21298

[53] Goodpaster, B.H., et al. (2005) Obesity, Regional Body Fat Distribution, and the Metabolic Syndrome in Older Men and Women. Archives of Internal Medicine, 165, 777-783. https://doi.org/10.1001/archinte.165.7.777

[54] Campbell, I.W. (2000) Antidiabetic Drugs Present and Future: Will Improving Insulin Resistance Benefit Cardiovascular Risk in Type 2 Diabetes Mellitus? Drugs, 60, 1017-1028. https://doi.org/10.2165/00003495-200060050-00004

[55] Gustafson, D., et al. (2003) An 18-Year Follow-Up of Overweight and Risk of Alzheimer Disease. Archives of Internal Medicine, 163, 1524-1528.

https://doi.org/10.1001/archinte.163.13.1524

[56] Naderali, E.K., Ratcliffe, S.H. and Dale, M.C. (2009) Obesity and Alzheimer's Disease: A Link between Body Weight and Cognitive Function in Old Age. American Journal of Alzheimer's Disease \& Other Dementias, 24, 445-449. https://doi.org/10.1177/1533317509348208

[57] Femminella, G.D. and Bencivenga, L., et al. (2017) Antidiabetic Drugs in Alzheimer's Disease: Mechanisms of Action and Future Perspectives. Journal of Diabetes Research, 2017, Article ID: 7420796.

[58] Shingo, A.S., Kanabayashi, T., Kito, S. and Murase, T. (2012) Intracerebroventricular Administration of an Insulin Analogue Recovers STZ-Induced Cognitive Decline in Rats. Behavioural Brain Research, 241, 105-111.

[59] Freiherr, J., et al. (2013) Intranasal Insulin as a Treatment for Alzheimer's Disease: A Review of Basic Research and Clinical Evidence. CNS Drugs, 27, 505-514. https://doi.org/10.1007/s40263-013-0076-8

[60] Landreth, G. (2007) Therapeutic Use of Agonists of the Nuclear Receptor PPAR $\gamma$ in Alzheimer's Disease. Current Alzheimer Research, 4, 159-164. https://doi.org/10.2174/156720507780362092

[61] Wang, L., Liu, W., Fan, Y., Liu, T.T. and Yu, C.J. (2017) Effect of Rosiglitazone on Amyloid Precursor Protein Processing and A $\beta$ Clearance in Streptozotocin-Induced Rat Model of Alzheimer's Disease. Iranian Journal of Basic Medical Sciences, 20, 474-480.

[62] Pancani, T., et al. (2009) Distinct Modulation of Voltage-Gated and Ligand-Gated $\mathrm{Ca}^{2+}$ Currents by PPAR- $\gamma$ Agonists in Cultured Hippocampal Neurons. Journal of Neurochemistry, 109, 1800-1811. https://doi.org/10.1111/j.1471-4159.2009.06107.x

[63] Harrington, C., et al. (2011) Rosiglitazone Does not Improve Cognition or Global Function when Used as Adjunctive Therapy to AChE Inhibitors in Mild-to-Moderate Alzheimer's Disease: Two Phase 3 Studies. Current Alzheimer Research, 8, 592-606. https://doi.org/10.2174/156720511796391935 
[64] Cheng, H.W., Shang, Y.P., Jiang, L., Shi, T.-L. and Wang, L. (2016) The Peroxisome Proliferators Activated Receptor-Gamma Agonists as Therapeutics for the Treatment of Alzheimer's Disease and Mild-to-Moderate Alzheimer's Disease: A Meta-Analysis. International Journal of Neuroscience, 126, 299-307. https://doi.org/10.3109/00207454.2015.1015722

[65] Alp, H., et al. (2012) Protective Effects of Beta Glucan and Gliclazide on Brain Tissue and Sciatic Nerve of Diabetic Rats Induced by Streptozosin. Experimental Diabetes Research, 2012, Article ID: 230342.

[66] Irons, B.K. and Minze, M.G. (2014) Drug Treatment of Type 2 Diabetes Mellitus in Patients for whom Metformin Is Contraindicated. Diabetes, Metabolic Syndrome and Obesity, 7, 15-24. https://doi.org/10.2147/DMSO.S38753

[67] Li, S.-N., Wang, X., Zeng, Q.T., Feng, Y.B., et al. (2009) Metformin Inhibits Nuclear Factor $\kappa \mathrm{B}$ Activation and Decreases Serum High-Sensitivity C-Reactive Protein Level in Experimental Atherogenesis of Rabbits. Heart Vessels, 24, 446-453. https://doi.org/10.1007/s00380-008-1137-7

[68] Rena, G., Pearson, E.R. and Sakamoto, K. (2013) Molecular Mechanism of Action of Metformin: Old or New Insights? Diabetologia, 56, 1898-1906.

https://doi.org/10.1007/s00125-013-2991-0

[69] Burcelin, R. (2014) The Antidiabetic Gutsy Role of Metformin Uncovered? Gut, 63, 706-707. https://doi.org/10.1136/gutjnl-2013-305370

[70] Chiang, M.-C., Cheng, Y.-C., Chen, S.-J., Yen, C.-H. and Huang, R.-N. (2016) Metformin Activation of AMPK-Dependent Pathways Is Neuroprotective in Human Neural Stem Cells against Amyloid-Beta-Induced Mitochondrial Dysfunction. EXperimental Cell Research, 347, 322-331. https://doi.org/10.1016/j.yexcr.2016.08.013

[71] DiTacchio, K. (2011) The Effect of AMPK Activation on Alzheimer's-Like Symptoms in APP Mice. University of California, San Diego.

[72] Herath, P.M., Cherbuin, N., Eramudugolla, R. and Anstey, K.J. (2016) The Effect of Diabetes Medication on Cognitive Function: Evidence from the PATH through Life Study. BioMed Research International, 2016, Article ID: 7208429

[73] Mostafa, D.K., Ismail, C.A. and Ghareeb, D.A. (2016) Differential Metformin Dose-Dependent Effects on Cognition in Rats: Role of Akt. Psychopharmacology, 233, 2513-2524. https://doi.org/10.1007/s00213-016-4301-2

[74] Hettich, M.M., et al. (2014) The Anti-Diabetic Drug Metformin Reduces BACE1 Protein Level by Interfering with the MID1 Complex. PLoS ONE, 9, e102420. https://doi.org/10.1371/journal.pone.0102420

[75] Alzoubi, K.H., Khabour, O.F., Al-azzam, S.I., Tashtoush, M.H. and Mhaidat, N.M. (2014) Metformin Eased Cognitive Impairment Induced by Chronic L-Methionine Administration: Potential Role of Oxidative Stress. Current Neuropharmacology, 12, 186-192. https://doi.org/10.2174/1570159X11666131120223201

[76] Arafa, N.M., Marie, M.A. and AlAzimi, S.A. (2016) Effect of Canagliflozin and Metformin on Cortical Neurotransmitters in a Diabetic Rat Model. Chemico-Biological Interactions, 258, 79-88. https://doi.org/10.1016/j.cbi.2016.08.016

[77] Abner, E.L., et al. (2016) Diabetes Is Associated with Cerebrovascular but not Alzheimer's Disease Neuropathology. Alzheimer's \& Dementia, 12, 882-889. https://doi.org/10.1016/j.jalz.2015.12.006

[78] Beeri, M.S., et al. (2005) Type 2 Diabetes Is Negatively Associated with Alzheimer's Disease Neuropathology. Journals of Gerontology. Series A, Biological Sciences and Medical Sciences, 60, 471-475. 
https://doi.org/10.1093/gerona/60.4.471

[79] Sonnen, J.A., Larson, E.B. and LBchB, K.B., et al. (2009) Different Patterns of Cerebral Injury in Dementia with or without Diabetes. Archives of Neurology, 66, 315-322. https://doi.org/10.1001/archneurol.2008.579

[80] Mima, Y., et al. (2016) Impact of Metformin on the Severity and Outcomes of Acute Ischemic Stroke in Patients with Type 2 Diabetes Mellitus. Journal of Stroke and Cerebrovascular Diseases, 25, 436-446.

https://doi.org/10.1016/j.jstrokecerebrovasdis.2015.10.016

[81] Giannarelli, R., Aragona, M., Coppelli, A. and Del Prato, S. (2003) Reducing Insulin Resistance with Metformin: The Evidence Today. Diabetes \& Metabolism, 29, 6S28-35.

[82] Hsu, C.-C., Wahlqvist, M.L., Lee, M.-S. and Tsai, H.-N. (2011) Incidence of Dementia Is Increased in Type 2 Diabetes and Reduced by the Use of Sulfonylureas and Metformin. Journal of Alzheimer's Disease, 24, 485-493. https://doi.org/10.3233/JAD-2011-101524

[83] Imfeld, P., Bodmer, M., Jick, S.S. and Meier, C.R. (2012) Metformin, Other Antidiabetic Drugs, and Risk of Alzheimer's Disease: A Population-Based Case-Control Study. Journal of the American Geriatrics Society, 60, 916-921. https://doi.org/10.1111/j.1532-5415.2012.03916.x

[84] Guo, M., et al. (2014) Metformin May Produce Antidepressant Effects through Improvement of Cognitive Function among Depressed Patients with Diabetes Mellitus. Clinical and Experimental Pharmacology and Physiology, 41, 650-656.

[85] McNeilly, A.D., Williamson, R., Balfour, D.J.K., Stewart, C.A. and Sutherland, C. (2012) A High-Fat-Diet-Induced Cognitive Deficit in Rats that Is Not Prevented by Improving Insulin Sensitivity with Metformin. Diabetologia, 55, 3061-3070. https://doi.org/10.1007/s00125-012-2686-y

[86] Qiu, W.Q. and Zhu, H. (2014) Amylin and Its Analogs: A Friend or Foe for the Treatment of Alzheimer's Disease? Frontiers in Aging Neuroscience, 6, 186. https://doi.org/10.3389/fnagi.2014.00186

[87] Verdile, G., Fuller, S.J. and Martins, R.N. (2015) The Role of Type 2 Diabetes in Neurodegeneration. Neurobiology of Disease, 84, 22-38. https://doi.org/10.1016/j.nbd.2015.04.008

[88] Adler, B.L., et al. (2014) Neuroprotective Effects of the Amylin Analogue Pramlintide on Alzheimer's Disease Pathogenesis and Cognition. Neurobiology of Aging, 35, 793-801. https://doi.org/10.1016/j.neurobiolaging.2013.10.076 